\title{
Isolement et caractérisation de la caséine kappa de chèvre
}

\author{
par \\ Louisette RAZANAJATOVO et Ch. ALAIS \\ Laboratoire de Biochimie Appliquée \\ Université de Nancy I, C.O. $140-$ F-54037 Nancy cedex
}

\section{INTRODUCTION}

Il existe des similitudes dans les propriétés, et notamment le comportement électrophorétique de la caséine du lait de divers ruminants, comme cela a été révélé par Alais et Jolles (1970). D'autres auteurs ont également mis en évidence des ressemblances entre la caséine de chèvre et la caséine de vache : Assenat (1967), Fox et O'Connor (1973). Cela suggère l'existence dans la caséine de chèvre de constituants de type $\alpha_{\mathrm{s}}, \beta$ et $\mathrm{K}$. Cependant, des différences apparaissent dans les analyses comparatives.

Au niveau des constituants les plus mobiles en milieu alcalin, Aschaffenburg (1968), Pierre et Portmann (1970) et Richardson et Creamer (1975) ont observé que le constituant de type $\alpha_{s}$ de la caséine de chèvre a une migration un peu plus lente que la caséine $\alpha_{\mathrm{si}}$ de vache, qui est le constituant rapide fortement prédominant. La caséine $\alpha_{\mathrm{s}}$ de chèvre semble correspondre à un constituant mineur, la caséine $\alpha_{s s}$ de vache ; elle contient deux résidus de cystéine par molécule de 25000 , alors que la caséine $\alpha_{\mathrm{S}_{1}}$ de vache est caractérisée par l'absence de cet acide aminé (Richardson et Creamer, 1975).

Des différences apparaissent aussi au niveau de la caséine $\beta$; chez la chèvre, c'est le constituant prédominant; elle présente la particularité d'être formée de deux bandes $\beta_{1}$ et $\beta_{2}$, la deuxième migrant de la même manière que la caséine $K$ de vache selon Richardson et Creamer (1974).

La position de la caséine $\mathrm{K}$ de chèvre est discutée. Sa migration électrophorétique en milieu alcalin ne permet pas de la distinguer car elle doit être cachée par les bandes $\beta$, comme cela a été observé 
par Zittle et Custer (1966) et Richardson et Creamer (1973). Ces derniers auteurs ont isolé la caséine $\mathrm{K}$ de chèvre par chromatographie sur échangeurs d'ions cellulosiques et noté que sa mobilité est plus grande que celle de la caséine $\mathrm{K}$ de vache ; en milieu acide, elle occupe une position intermédiaire entre les bandes $\beta$ et $\alpha_{s}$; il ne semble pas exister de variations génétiques.

Des travaux effectués à Surgères par le Groupe de Recherche de Biochimie Appliquée de Nancy ont amorcé l'identification de la caséine $\mathrm{K}$ en vue d'un phénotypage. Cette identification a été faite par la méthode de précipitation au calcium et le spectre électrophorétique général obtenu sur les 96 caséines étudiées montre trois bandes d'inégale intensité dans la région $\beta$. L'ordre de migration des trois constituants n'était pas déterminé.

Dix échantillons de laits individuels provenant de chèvres homozygotes nous ont été ramenés de Surgères, auxquels nous avons joint des échantillons de la région de Nancy. Avec ce matériel, nous avons voulu améliorer nos connaissances sur l'identification de la caséine $\mathrm{K}$ de chèvre et nous avons, dans ce but, effectué des fractionnements de la caséine de ces laits par purification et par chromatographie d'affinité.

\section{MATERIEL ET METHODES}

\section{Lait}

Le lait de chèvre en pleine lactation provenait du troupeau de la ferme expérimentale de l'ENSAIA de Nancy (six échantillons) et de la ferme de J. P. Massignac, Saint-Christophe (17 - La Jarrie).

La caséine $\mathrm{K}$ a été isolée de plusieurs manières, en se basant sur les travaux consacrés à la caséine $\mathrm{K}$ de vache.

\section{Fractionnement par précipitation}

Le schéma de la figure 1 montre les étapes de la purification. Nous avons tout d'abord effectué une simple précipitation en milieu urée par les ions calcium, selon Waugh et V. Hippel (1956), pour obtenir rapidement « la fraction $K$ » du surnageant (A).

Une purification complémentaire, selon Mac Kenzie et Wake (1961) a été appliquée à la fraction précédente, par précipitation au sulfate de sodium et à l'acétate d'ammonium dans l'éthanol $\left(\mathrm{B}_{1}\right)$. Un fractionnement parallèle à partir de la caséine entière par la méthode à l'urée et à l'acide sulfurique de Zittle et Custer (1963) a été ensuite pratiqué avec un complément de purification à l'acétate d'ammonium comme ci-dessus $\left(\mathrm{B}_{2}\right)$. Cette dernière fraction a été retraitée, en vue d'éliminer les traces de fraction $\alpha_{\mathrm{s}}$ et $\beta$, par l'urée et le métaphosphate selon Zittle et Custer (1966) (C). 


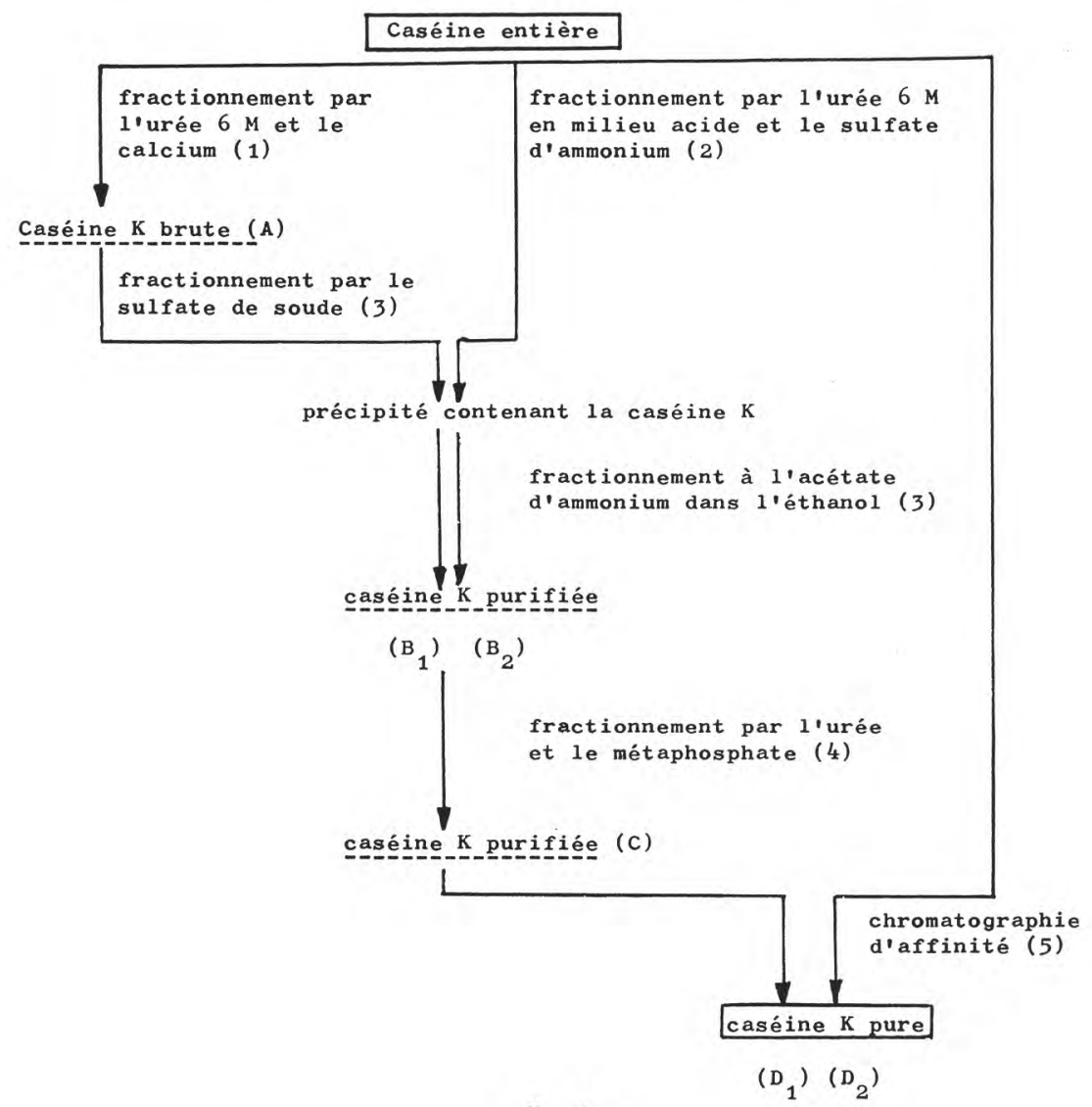

fig. 1

Schéma de purification de la caséine de chèvre

(1) Selon Waugh et von Hippel (1956) ; (2) Mac Kenzie et Wake (1961) ;

(3) Zittle et Custer (1963) ; (4) Zittle et Custer (1966) ; (3) Klostermeyer et Nijhuis (1975).

\section{Chromatographie d'affinité}

Nous avons suivi la méthode utilisée par Klostermeyer et Nijhuis (1975) pour isoler la caséine K de vache et nous l'avons appliquée à la fois à la caséine entière et à la caséine $K(C)$. Le support est le Thiol-Sépharose $4 \mathrm{~B}$ (Pharmacia) qui retient les constituants protidiques contenant des résidus de cystéine par formation de ponts disulfures ; les autres constituants traversent directement la colonne dans la phase circulante. Ainsi, les caséines $\alpha_{\mathrm{S} 1}$ et $\beta$ sont éliminées. 
La caséine $\mathrm{K}$ et les constituants mineurs de type $\alpha_{\mathrm{ss}}$ sont retenus ; ils sont élués par passage de L-cystéine avec la mercaptopyridine. Avant la chromatographie, la caséine entière est traitée par un agent réducteur, le dithiothréitol, qui libère les groupes $\mathrm{SH}$.

\section{Analyses}

Les examens électrophorétiques ont été effectués en gel d'amidon

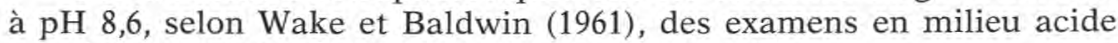
ont été également faits.

Les fractions $\mathrm{K}$ ont été, de plus, caractérisées par leur teneur en glucides et par leur sensibilité à la chymosine. Le dosage des oses neutres a été fait selon Rondle et Morgan (1955).

L'action de la chymosine a été étudiée sur des solutions à 2 p. 100 à $32^{\circ} \mathrm{C}$, avec $0,05 \mathrm{UP} / \mathrm{ml}$ d'enzyme ; après $30 \mathrm{mn}$ d'action on a dosé l'azote non protéique (NPN) selon Alais (1956).

\section{RESULTATS}

\section{Examens électrophorétiques de la caséine entière de chèvre}

La figure 2 présente le diagramme d'électrophorèse de deux échantillons de caséine individuelle $(\mathrm{a}, \mathrm{b})$ avec comme point de comparaison, la caséine entière de vache (v). On voit qu'il n'y a pas de bande au niveau de $\alpha_{s_{1}}$, dans la caséine de chèvre, mais une bande assez faiblement colorée au niveau de $\alpha_{s s}$. Dans la zone moyenne on observe trois bandes nettes, deux fortes et une faible, la plus lente migrant au niveau de la caséine $\beta$ de vache; on n'observe pas de bande lente (au niveau de la caséine K). Entre les bandes $\alpha_{s}$ et $\beta$ on observe trois bandes mineures. On a remarqué que les diagrammes sont très réguliers d'un échantillon à l'autre.

\section{Isolement de la caséine}

La simple précipitation par le calcium laisse subsister un peu de caséine $\alpha$ s dans la fraction $K$ (A) (fig. 2, c, d). Parmi les trois bandes de la zone $\beta$, on observe que la plus rapide, qui auparavant était la plus faible, est devenue aussi forte ou plus forte que les deux suivantes ; cette bande correspond probablement à la caséine $\mathrm{K}$, les deux autres étant $\beta_{1}$ et $\beta_{2}$. La purification complémentaire par traitement à l'acétate et à l'éthanol n'améliore pas considérablement la pureté. Par contre, la précipitation par le sulfate d'ammonium réduit beaucoup les bandes $\beta_{1}$ et $\beta_{2}$ et accroît la bande $K$. La dernière précipitation au métaphosphate élimine les bandes $\alpha_{\mathrm{s}}$ et rend nettement plus visible la bande la plus rapide de la zone $\beta$ (fig. 2 , e, f). 


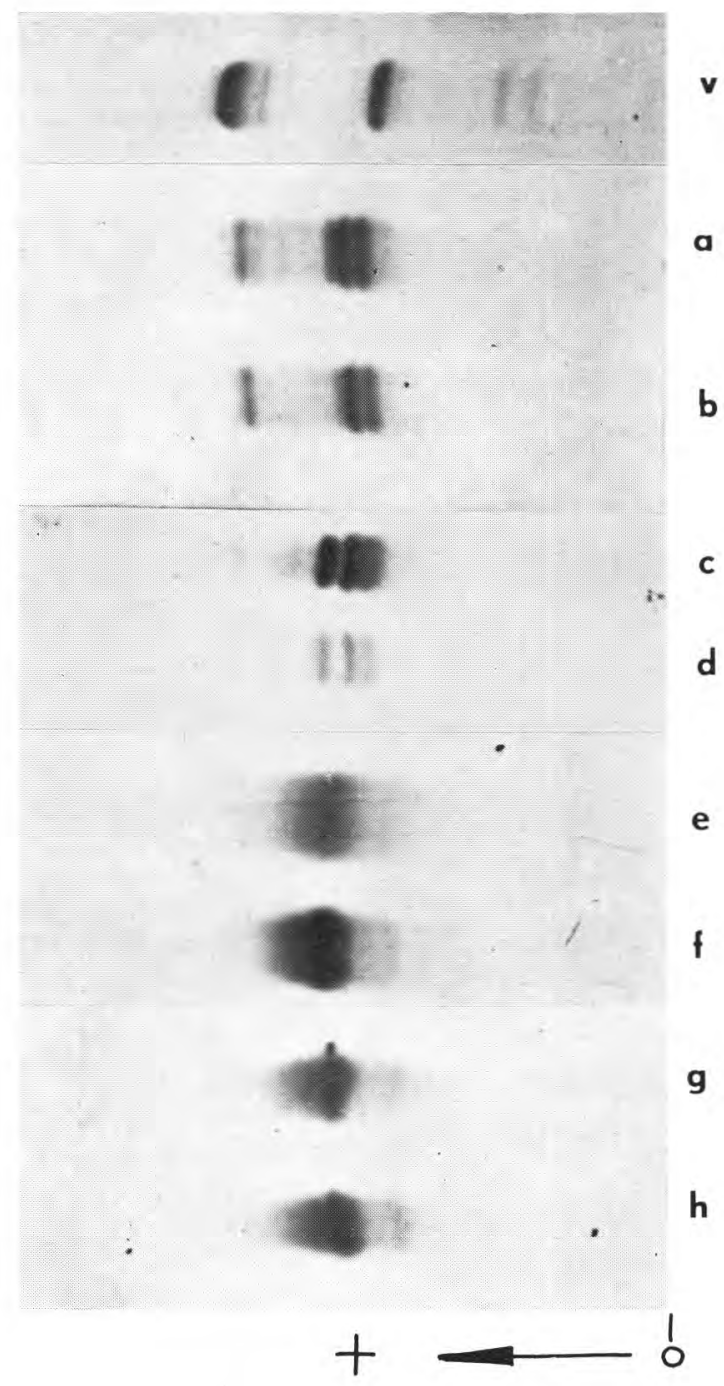

fig. 2

Electrophorèse en gel d'amidon $\mathrm{pH}$ 8,6 de la caséine de chèvre $(\mathrm{a}, \mathrm{b})$, de la fraction $\mathrm{K}$ brute $(\mathrm{c}, \mathrm{d})$, de la caséine $\mathrm{K}$ de chèvre fractionnée par le métaphosphate $(e, f)$, de la caséine $K$ de chèvre séparée par chromatographie d'affinité $(\mathrm{g}, \mathrm{h})$ et de la caséine entière de vache $(v)$. 


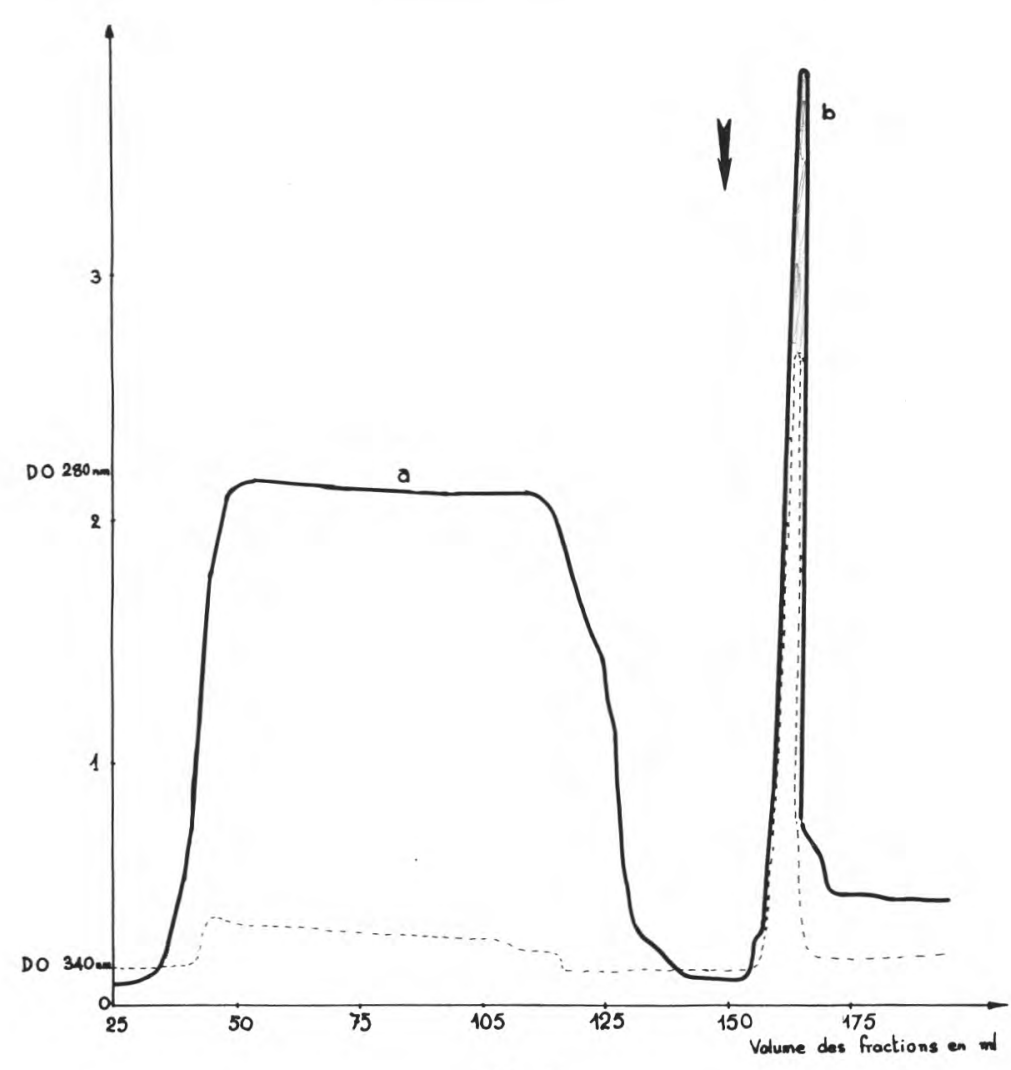

fig. 3

Diagramme d'élution de la chromatographie d'affinité de la caséine entière de chèvre. Premier tampon d'élution : Tris-HCl, $\mathrm{pH} 7$; deuxième tampon d'élution : Tris-HCl, pH 8 + cystéine (à la flèche); colonne $40 \times 1$, débit $25 \mathrm{ml} / \mathrm{h}$, fractions de $3 \mathrm{ml}$. Absorption à $280 \mathrm{~nm}$ (caséine) ; absorption à $340 \mathrm{~nm}$ (2-mercaptopyridine).

La chromatographie d'affinité a été appliquée à cinq échantillons dont trois de la caséine entière de chèvre et deux de la caséine $K$ purifiée. La figure 3 présente un diagramme d'élution avec une caséine entière. Le gros pic « a » en forme de plateau irrégulier, représente la sortie des caséines non retenues sur le Thiol-Sépharose ; le pic «b », élué par la cystéine, correspond à la caséine K. Les diagrammes d'électrophorèse de la figure $2, \mathrm{~g}, \mathrm{~h}$, montrent que cette deuxième fraction est presque homogène ; le premier pic ne le renferme pas. Lorsque l'on chromatographie les caséines $\mathrm{K}$ purifiées, on a le même profil d'élution, mais le pic « a » est réduit. 


\section{Caractérisation chimique}

Les résultats du tableau 1 montrent que les fractions $\mathrm{K}$ sont beaucoup plus riches en oses neutres, osamines et acide sialique que la caséine entière dont elles proviennent.

En se référant à la teneur de glucides connus dans la caséine $\mathrm{K}$ de vache selon Alais (1974) nous pouvons considérer que la fraction analysée correspond à la fraction $\mathrm{K}$.

D'autre part, après l'action de la chymosine, les valeurs de NPN libéré de la caséine $\mathrm{K}$ de chèvre sont très élevées (tab. 2), par rapport aux valeurs obtenues avec la caséine entière. Ceci montre

\section{$T A B L E A U 1$}

Composition glucidique de la caséine entière de chèvre et des fractions $\mathrm{K}$

\begin{tabular}{|c|c|c|c|c|}
\hline & & Oses neutres & Osamines & acide sialique* \\
\hline Caséine entière & $\begin{array}{l}\mathrm{m} \\
\mathrm{n} \\
\sigma\end{array}$ & $\begin{array}{c}0,38 \\
16 \\
0,015\end{array}$ & $\begin{array}{c}0,30 \\
16 \\
0,026\end{array}$ & $\begin{array}{c}0,13 \\
16 \\
0,003\end{array}$ \\
\hline Caséine $K$ purifiée $\left(B_{1}, B_{2}\right)$ & $\begin{array}{l}\mathrm{m} \\
\mathrm{n} \\
\sigma\end{array}$ & $\begin{array}{c}1,27 \\
17 \\
0,023\end{array}$ & $\begin{array}{c}1,67 \\
17 \\
0,02\end{array}$ & $\begin{array}{c}1,97 \\
17 \\
0,026\end{array}$ \\
\hline Caséine $\mathrm{K}$ purifiée $(\mathrm{C})$ & $\begin{array}{l}\mathrm{m} \\
\mathrm{n} \\
\sigma\end{array}$ & $\begin{array}{c}1,29 \\
11 \\
0,012\end{array}$ & $\begin{array}{c}1,69 \\
11 \\
0,013\end{array}$ & $\begin{array}{c}2,01 \\
11 \\
0,038\end{array}$ \\
\hline Caséine $\mathrm{K}$ pure $(\mathrm{D})$ & $\begin{array}{l}\mathrm{m} \\
\mathrm{n} \\
\sigma\end{array}$ & $\begin{array}{c}1,34 \\
4 \\
0,01\end{array}$ & $\begin{array}{c}1,75 \\
4 \\
0,013\end{array}$ & \\
\hline
\end{tabular}

* N-acétyl-neuraminique.

TABLEAU 2. - Protéolyse par la chymosine

\begin{tabular}{c|c|c|c}
\hline \multicolumn{1}{c|}{ Caséine } & Entière & K - B & K - D \\
\hline & 0,63 & 1,40 & 1,56 \\
NPN-O p. $100 \mathrm{Nt}$ & 8,93 & 10,44 \\
Augmentation NPN p. $100 \mathrm{Nt}$ & 0,74 & & \\
\hline
\end{tabular}


sa sensibilité à la chymosine. Au cours de ces dosages, les valeurs maximales ont été obtenues avec la caséine $\mathrm{K}$ issue de la chromatographie d'affinité (D).

\section{DISCUSSION - CONCLUSION}

Les quatre méthodes de précipitation utilisées ont été élaborées pour l'isolement de la caséine $\mathrm{K}$ de vache. Or, au cours de leur application à la caséine de chèvre, nous avons observé par électrophorèse un renforcement progressif de la bande rapide de la zone $\beta$, ce qui nous amène à penser que cette bande constitue la caséine $\mathrm{K}$ de chèvre. Ceci est confirmé par l'application de la chromatographie d'affinité ; avec la fraction éluée par la cystéine, nous n'observons que cette bande caractéristique dans la région moyenne ; une faible bande persiste au niveau de $\alpha_{\mathrm{s}}$. Selon Richardson et Creamer (1975) la caséine $\alpha_{s}$ de chèvre, contrairement à celle de la vache, contiendrait de la cystéine et correspondrait en fait au constituant mineur $\alpha_{s_{3}}$ de la caséine bovine. Mais si toute la caséine $\alpha_{s}$ de chèvre était porteuse, après réduction au dithiothréitol, de groupes $\mathrm{SH}$, elle aurait dû être retenue sur le Séphadex et éluée par la L-cystéine, avec la caséine $\mathrm{K}$. Il semble qu'il n'en soit pas ainsi, vu la très faible bande électrophorétique observée ; ou bien les ponts S-S de la caséine $\alpha_{s}$ de chèvre ne sont que partiellement rompus dans ces conditions, ou bien cette caséine $\alpha_{s}$ est hétérogène et un constituant seulement contient de la cystéine. Malgré la présence en faible proportion de caséine $\alpha$, nous pouvons affirmer que cette bande rapide représente la caséine $\mathrm{K}$ de chèvre, ce qui implique que la caséine $\beta$ de chèvre migre avant les bandes $\beta$, contrairement à celle de la vache, par électrophorèse à $\mathrm{pH} 8,6$.

D'autre part, nous n'observons pas de bandes annexes à la bande proprement dite, le calcul des références frontales de cette bande donne des valeurs constantes de 0,86 quel que soit l'échantillon (au total 16) ; la caséine $\mathrm{K}$ occupe donc une position invariable. Nous rejoignons ainsi l'affirmation de Richardson et Creamer sur l'absence de polymorphisme génétique de la caséine $\mathrm{K}$.

Les différentes fractions $\mathrm{K}$ obtenues présentent des propriétés caractéristiques de la caséine $\mathrm{K}$ du fait de sa forte teneur en glucides et un taux élevé de NPN libéré par la chymosine, par rapport à la caséine entière (tab. 2). Signalons en particulier la valeur obtenue avec la fraction $D_{1}, D_{2}$ qui est très forte, ce qui est l'indication d'une pureté élevée.

$\mathrm{Au}$ terme de notre étude, nous prenons connaissance du récent article de Kotts et Jenness (1976) dans lequel ils montrent que la première bande de la zone moyenne est bien la caséine $\mathrm{K}$ de chèvre car elle disparaît sous l'action de la présure, ce qui confirme nos résultats. 


\section{Ré s u m é}

La caséine $\mathrm{K}$ de chèvre a été isolée par quatre méthodes de précipitation, puis par chromatographie d'affinité utilisant le ThiolSépharose $4 \mathrm{~B}$ comme support. Les fractions obtenues ont été caractérisées par électropborèse en gel d'amidon à $\mathrm{pH} 8,6$. La caséine $\mathrm{K}$ a donc été ainsi identifiée comme migrant en avant des caséines $\beta$; elle occupe une position invariable. La caséine $\mathrm{K}$ de chèvre présente les mêmes caractéristiques générales que celle de la vache.

\section{S u $\mathbf{m} \mathbf{m}$ a r y}

\section{ISOLATION AND CHARACTERIZATION OF GOAT K-CASEIN}

The fractionation of goat casein by salt precipitation or by affinity chromatography on Thiol-Sepharose had shown the presence of a K-component similar to cow K-casein but migrating ahead $\beta$-casein in starch-gel electrophoresis at alcaline $\mathrm{pH}$. The electrophoretical behavior of the goat $\mathrm{K}$-casein is now well defined; it contains glucides in rather high proportions and it is chymosin sensitive.

\section{Remerciements}

Nous remercions $M$. Massignac et le Service de Zootechnie de I'E.N.S.A.I.A. de Nancy pour la fourniture de laits de chèvre.

Nous remercions aussi l'Ecole Nationale d'Industrie laitière pour les facilités accordées pour le phénotypage.

\section{Bibliographie}

Alais (C.), Jolles (P.) (1970). - Intern. J. Biochem., 1, 546.

AlaIs (C.) (1956). - XIV Congr. Intern. Lait, 2, 823-837.

Alais (C.) (1974). - Chimica, 28, 597.

Assenat (L.) (1967). - Le Lait, 47, chap. III, 393.

AschaffenduRg (R.) (1968). - J. Dairy Research, 35, 383.

Fox (P. F.), O'ConNoR (P.) (1973), - Neth. Milk Dairy, 27, 199-217.

Klostermeyer (H.), NiJHUIS (H.) (1975). - Milchwissenschaft, 9, 30.

KotTs (C.), JenNesS (J.) (1976). - J. Dairy Sci., 59, 816-822.

Mac Kenzie (H. A.), WaKe (R. G.) (1961). - Biochim. Biophys. Acta, 47, 240.

Pierre (A.), Portmann (1970). - Ann. Technol. Agric., 19, 107-130.

Richardson (B. C.), Creamer (L. K.) (1973). - Biochim. Biophys. Acta, 310, 1, 111-117.

Richardson (B. C.), Creamer (L. K.) (1974). - Biochim. Biophys. Acta, 365, 1 , 133-137.

Richardson (B. C.), Creamer (L. K.) (1974). - XIX Int. Dairy Congress IE, 268.

Richardson (B. C.), CREAMER (L. K.) (1974). - J. Dairy Res., 41, 2, 239-247.

Richardson (B. C.), Creamer (L. K.) (1975). - Biochim. Biophys. Acta, 393, 37-47.

Rondle (J.), Morgan (W, T. J.) (1955). - Biochem. J., 61, 586.

Waugh (D. F.), Von Hippel (P. H.) (1956). - J. Amer. Chem. Soc., 78, 4576-4582.

Wake (C.), Baldwin (R. L.) (1961). - Biochim. Biophys. Acta, 47, 225-239.

ZitTle (C. A.), Custer (J. H.) (1966). - J. Dairy Sci., 6, 706-707.

Zitrle (C. A.), Custer (J. F.) (1966). - J. Dairy Sci., 49, 788-791.

ZitTle (C. A.), Custer (J. H.) (1963). - J. Dairy Sci., 46, 1183. 